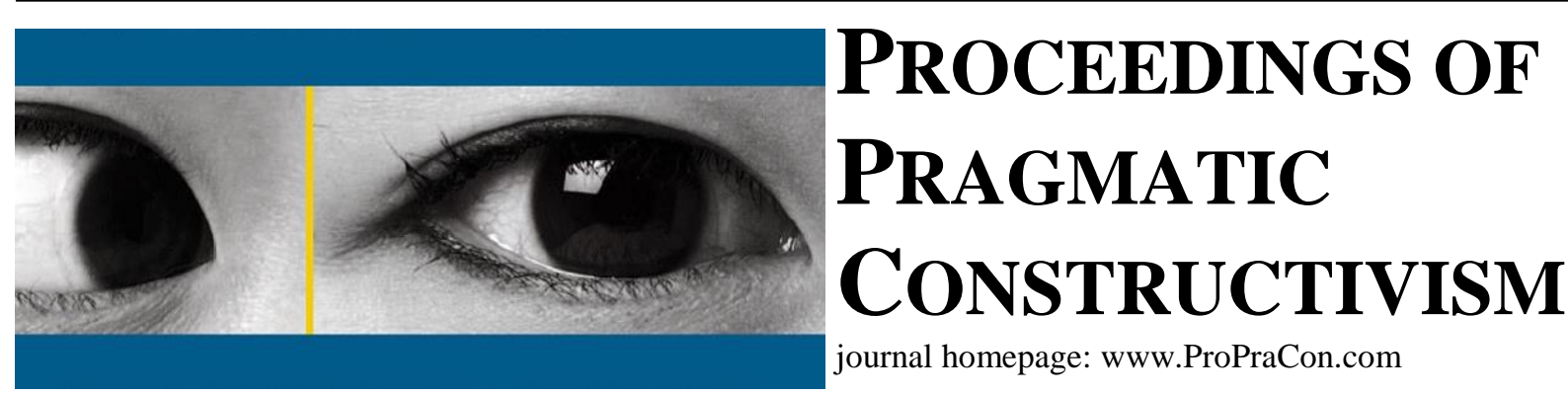

\title{
Cost management and Big Data - searching for possibilities
}

\author{
Morten Jakobsen \\ Department of Management, Aarhus University, Denmark. \\ E-mail: mja@mgmt.au.dk
}

Cost management is a central aspect of managing an organisation. Costs are a monetary representation of the resource spent to provide a product or service for a sale in a market. These resources have a scarcity that must be taken into account. This is done by choosing the utilisation of resources in a way that is found will lead to the most profitable outcome.

Cost as phenomenon is not static. Static in the sense that a certain event will lead to certain costs, and that costs thereby can predicted out of certain events. Costs are consequences of specific responses to an event. The specific response is the often chosen from a number of possible responses to the specific event. These possible responses typically consists of a number of resources that in combination provides a possible response to an event. Doing cost accounting for each possible response is in itself a complicated affair that requires craftsmanship and business knowledge. What further complicates this decision process is that the cost per resource unit may change depending on what alternative we choose. The alternatives are not only to be made among the possible responses, but may also include selling the resource at net realisable value, and taking into account what the replacement cost of the resource is, if we want to replace the resource. These economic approaches point into the future and as such in the same direction as decision making. Unfortunately, these economic perspectives tend to explode in the hands of decision makers and complicate decision making to an extent that is practically unacceptable. This is probably why much decision making is based on accounting data, where the cost estimates of resources are based on the historic value of the resources, for instance the purchase price of the raw material at stock. Such an approach is convenient because the data is in most accounting systems at hand. In addition such fact-based data has high degree of reliability leaving less room for discussions concerning the data input to the decision making process. However, these aspects do not remove the point that basing decision making on historic cost is like driving a car by looking at the rear-view mirror. It is possible, but only as long as the road forward is similar to the road covered.

The first obstacle towards including costs that are future oriented seem to be the availability of data. The phenomenon Big Data is claimed to be able to deliver data of different kinds. Potentially such data may provide the foundation for cost management decisions. However, the nature of Big Data is not structured as traditional accounting data. Hence the validity of Big Data may be smaller and the usage of such data has to be made with caution, because the Big Data may not live up to the criteria that traditionally has been set for cost data meant or decision making. Concerning reliability of cost data, the usage of Big Data will probably also create matters that has to be addressed. Since Big Data is not structured according to a certain logic, the patterns found from Big Data is likely to be highly dependent upon the search process. The result can therefore not be included in the decision process at face value but somehow needs to undergo some kind of interpretation and validation.

The objective of this paper is to discuss how cost management can embrace Big Data and use such source of information as input for forward looking cost management decisions without jeopardizing traditional cost management criteria.

The paper is structured as follows. First it discusses the concept cost management and the criteria that the profession traditionally has used as guidance for cost management practice. Second the paper discuss the phenomenon of big data. Third the paper discuss how cost management can embrace big data. This discussion is made within the framework pragmatic constructivism. 